\title{
Measurement of Water ball Lens Transmittance
}

\author{
Haiyan Yang ${ }^{1, a, *}$, Xiaopan Li ${ }^{1, b}$, Jiufu Chen ${ }^{1, c}$, Peng Ai ${ }^{1, d}$, Guofang Du ${ }^{1, \mathrm{e}}$ Bo Tang ${ }^{2, \mathrm{f}}$ and \\ Chunxue $Y u^{1, g}$ \\ ${ }^{1}$ School of Physics and Information Engineering, Zhaotong University, Zhaotong 657000, China; \\ ${ }^{2}$ Qujing No.1 Middle School Qiling Campus,Qujing 655000,China; \\ aelaincoco@foxmail.com, ${ }^{b}$ ztu_lxp@foxmail.com, ${ }^{c} 403812462 @ q q . c o m,{ }^{d} 15808710114 @ 139 . c o m,{ }^{e} 1057988$

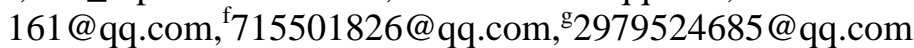

Keywords: water ball lens, high-pressure response, transmittance, wide-angle lens

\begin{abstract}
Water ball lens refer to a simple thick convex lens made of glass spherical shell filled with highly pure water. Besides, the image of water ball lens is not a plane, but half of the spherical image with the photo-center as the center and effective focal length as the radius. Water ball lens have good symmetry of the ball, and the detection of each direction is consistent. The imaging performance of water ball lens is the focus of this paper. In this paper, through the test of the water ball lens with a diameter of $35 \mathrm{~cm}$, the transmittance of the water ball lens under the measurement conditions is given.
\end{abstract}

\section{Introduction to the Test System}

The experiment was carried out in a long, dark chamber, using a water ball lens of $35 \mathrm{~cm}$ in diameter and a glass thickness of about $0.4 \mathrm{~cm}$ filled with high purity water. With LED as the light source, the LED generates a fixed frequency and waveform pulse from the signal generator. PMT is taken as a photodetector, and make the center of the LED, water ball lens and photomultiplier tube on the same line. The entire test system also includes NIM chassis, CAMAC chassis, high-voltage power supply, HP produced 8130A signal generator. Among them, the 8130A voltage maximum output amplitude is $5.2 \mathrm{~V}$, and the minimum resolution is $10 \mathrm{mV}$; the minimum resolution of the pulse width is 10ps. LRS 2249A is adopted in ADC, the resolution is $0.25 \mathrm{pC} /$ count and the range 256pC. ET9829B is used for PMT. As shown in Figure 1

\section{Photomultiplier Tube Performance Test}

It is tested with British ET Corporation 9829B, PMT diameter is 5.2cm (2 inches), and the glass window is a thin dome, as shown in Figure 2. The cathode material is bialkali, with 12 beryllium copper poles. 9829B provides the design of two voltage dividers A and B, as shown in Figure 3. In order to obtain better pulse linearity, this paper selects the divider B partial pressure method. Single photoelectron peaks are a basic parameter that characterizes PMT performance. LED is used as light source for single photon peak measurement. LED is generated by pulse signal generated by signal generator. The choice of high voltage at the time of measurement should ensure that the PMT gain is large and the intensity adjustment is from being weak to being strong to distinguish the signal and noise, and then gradually weaken until the ADC takes about $90 \%$ of the time to measure the step, thus ensuring that most of the signals are a single photoelectron signal. Because according to Poisson formula

$$
P(n)=\frac{e^{-\mu} \mu^{n}}{n !}
$$




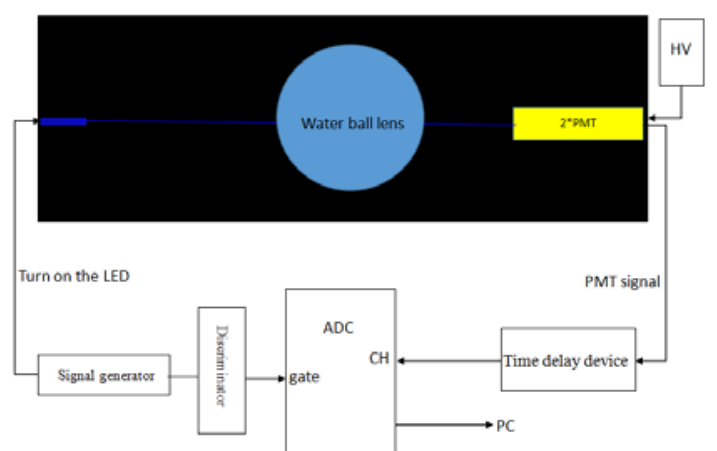

Fig. 1. Test Device Diagram

In formula (1), $\mathrm{P}(\mathrm{n})$ is the possibility of detecting $\mathrm{n}$ optoelectronics, and $\mu$ is the average value of the photoelectron arriving at the first pole. Assuming that $90 \%$ of the time does not detect the signal, then the possibility for no probability of photoelectrons is $\mathrm{P}(0)=e^{-\mu}=0.9$, so $\mu=0.105$. Take $\mu=0.105$ into formula (1) to obtain the possibility of seeking the single photoelectron $\mathrm{P}(1)=\mu e^{-\mu}=0.095$, so $\mathrm{P}(\mathrm{n}>1)=1-\mathrm{P}(0)-\mathrm{P}(1)=1-0.9-0.095=0.005$. That is to say at this time, the detections are mainly single photoelectrons. Figure 4 is a single photoelectron peak measured at $1600 \mathrm{~V}$ at 160029 . The fitting peak is the absolute gain under this condition $7.22 \mathrm{e}+06$.
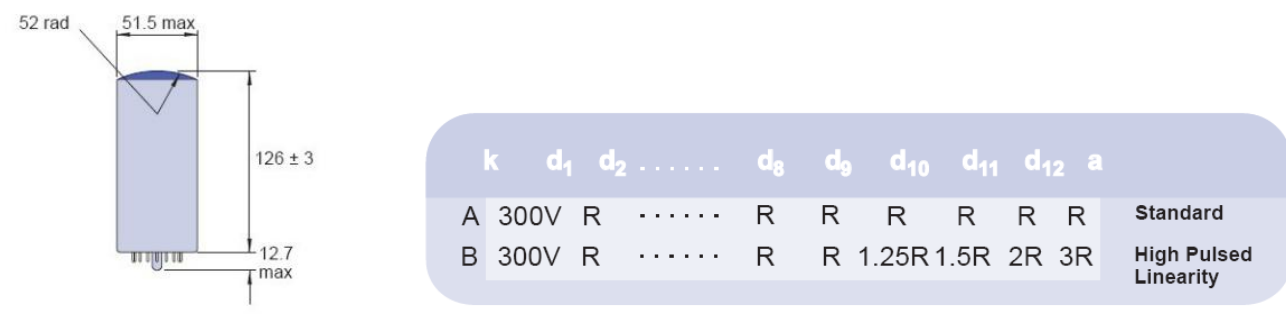

Fig. 2. (on left)9829B Outline Parameters Diagram

Fig. 3. (on right)Two Partial Pressure Modes of 9829B

The relationship between PMT high pressure and the gains satisfies $G=k V^{\beta}$, i.e., the gain $\mathrm{G}$ changes with the $\beta$ times of the high pressure. 。 Figure 5 is the laboratory measured 9829B high pressure response curve, with good linearity, fitted to get the $\beta$ value is 9.829 .
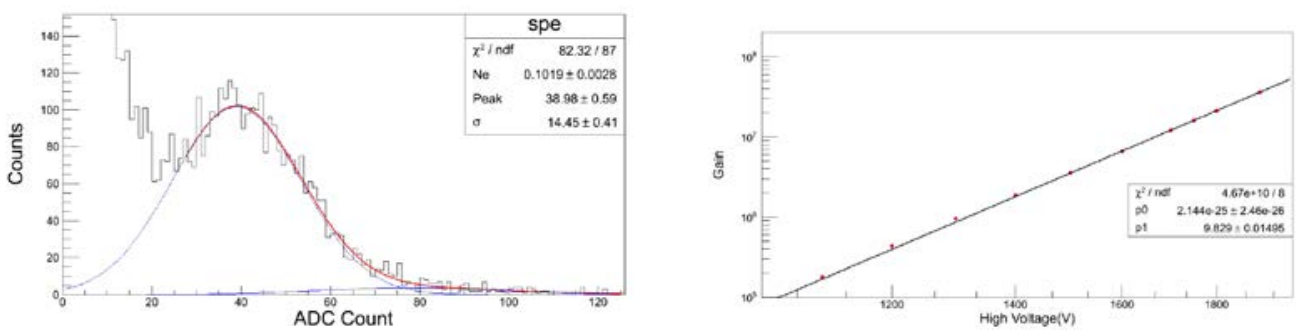

Fig 4. (on left)A single photoelectron peak under high pressure of 49829B1600V

Fig. 5.(on right) Response Curve under 9829B High Pressure

\section{Water ball Lens Transmittance Test Results}

As the light propagates in the medium when the intensity will be attenuated with the propagation distance, light will interact with air, glass and water when going through the water ball lens, and light absorption, scattering and dispersion will occur. The light that reaches the surface of the water ball lens cannot pass through the water ball lens

Table 1. Basic Performance Parameters Given in PMTET9829B Manual without loss of arrival. The wide-angle lens requires that the transmittance of the lens should be better than $70 \%$. This experiment verifies that the transmittance of the water ball lens meets the requirements of the wide-angle lens.

Assuming the light intensity emitted by the laser LED is the unit 1, the transmittance of the water 
ball lens is defined in the paper as that when the laser LED is taken as the light source, the distance between the LED and the PMT is constant, the laser light reaches the PMT light intensity is $\mathrm{I}_{1}$, and the PMT light intensity is $\mathrm{I}_{2}$ after the air reaches water ball lens, and then the water ball lens transmittance $t$ is

$$
t=\frac{I_{2}}{I_{1}} \times 100 \%
$$

Test the PMT light intensity when there are the convex lens and there is no convex lens, as shown in Fig.6. The transmittance of the water ball lens can be calculated according to (2). When the incident parallel light covers the entire sphere, the transmittance cannot be accurately measured, because the general PMT receiving area is limited and cannot cover the entire image. Influenced by the water ball lens spherical effect, there will be part of the light deflection to the PMT to accept the outside, which results in inaccurate measurement results. Therefore, a laser LED is chosen in the experiment as a light source, the collimation of good and small luminous area, the laser by the water ball lens spherical zero incidence, which can ensure that the PMT detects almost all of the transmitted light. LED series $50 \Omega$ resistor is about $76 \mathrm{~cm}$ from the center of the ball, PMT is put on the core from the center of about $40 \mathrm{~cm}$ effective focal plane for measurement. Figure 7 shows the test results, no lens (blue curve shown) measured the number of ADC Road, 269.95 Road, there are water ball lens (red curve) measured ADC channel number is 228.09 Road. Therefore, the transmittance is

$t=\frac{228.09}{269.95} \times 100 \% \approx 82 \%$

The measurement results show that the transmittance of the water ball lens satisfies the requirements of the wide angle lens.
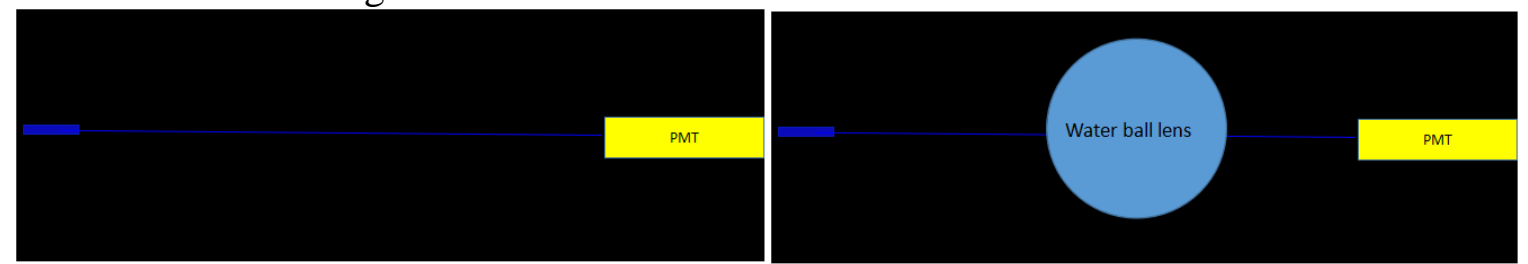

Fig. 6 Diagram of Testing Water ball Lens’ Transmittance

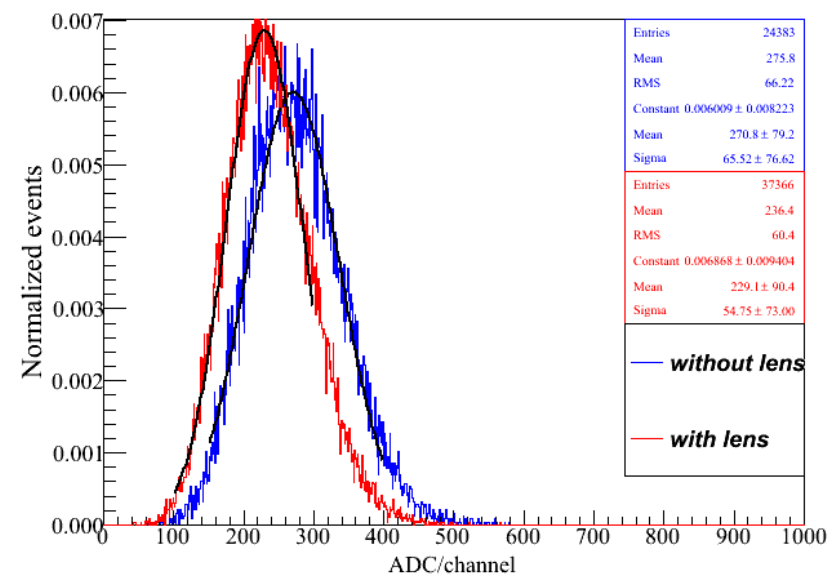

Fig. 7. Signal Intensity Curve in the presence or absence of convex lens in PMT measurement

\section{References}

[1] Xie Yigang et al. Particle Detector and Data Acquisition. Science Press, Beijing,2003: 259.

[2] A. M. Hillas. J. Phys, 16(1990), 1271-128.

[3] F. Aharonian et al. Rep. Prog. Phys., 71(2008)

[4] A. Horibe, M. Baba, E. Nihei, and Y. Koike, "High Efficiency and High Visual Quality LCD Backlighting System", SID, 12(1998), 153. 
[5] P.N.Murgatroyd, Theory of space-charge limited current enhancd by frenkel effect, J.Phys.D: AppI.Phys, 3(1970),151. 